\title{
NEIGHBORHOODS AND PARTIAL SUMS OF MEROMORPHIC UNIVALENT FUNCTIONS
}

G. Murugusundaramoorthy* and S.V.S. Velayudam**

\begin{abstract}
In this paper we determine neighborhood results and partial sums for certain class of meromorphic univalent functions with positive coefficients defined by Ruscheweyh derivatives.
\end{abstract}

2000 Mathematics Subject Classification: 30C45.

Keywords and Phrases: Meromorphic, neighborhood, convolution, partial sums.

\section{Introduction}

Let $M$ denotes the class of functions of the form

$$
f(z)=\frac{1}{z}+\sum_{k=1}^{\infty} a_{k} z^{k}
$$

* Department of Mathematics, Vellore Instifute of Technology, Deemed University, Vellore-632014, India. email: gmsmoorthy@yahoo.com.

** Department of Mathematics, M.N.M. Jain Engineering College. Thorappakam, Chennai - 600 096, India. 
which are regular and univalent in the punctured disc $E=\{z: 0<|z|<1\} A$ function $f(z)$ belonging to $M$ is said to be meromorphically starlike of order $\alpha$ if it satisfies

$$
\operatorname{Re}\left\{\frac{-z f^{\prime}(z)}{f(z)}\right\}>\alpha
$$

for some $\alpha(0 \leq \alpha<1)$ and all $z \in U=\{z:|z|<\}$. We denote $M(\alpha)$ the class of all meromorphically starlike functions of order $\alpha$. The class $M(\alpha)$ and related classes have been extensively studied in $[2,5,6,8]$.

Let $M(\alpha, \lambda, A, B)$ denote the class of functions $f$ in $M$ satisfying the condition

$$
\left|\frac{z^{2}\left(D^{\lambda} f(z)^{\prime}+1\right.}{B z^{2}\left(D^{\lambda} f(z)\right)^{\prime}+A}\right|<\alpha
$$

for some $\alpha>0,-1 \leq B<A \leq 1$ and $|B \alpha| \leq 1$ and for all $z \in U$, where $D^{\lambda}: M \rightarrow M$ is the operator defined by

$$
D^{\lambda} f(z)=\frac{1}{z(1-z)^{\lambda+1}} * f(z),(\lambda>-1)
$$

From the identity

$$
\frac{1}{z(1-z)^{\lambda+2}}=\frac{1}{z(1-z)^{\lambda+1}} *\left(\frac{\lambda+2}{z(1-z)(\lambda+1)}+\frac{2 z-1}{z(1-z)^{2}(\lambda+1)}\right) .
$$

We get

$$
z\left(D^{\lambda} f(z)\right)^{\prime}=(\lambda+1) D^{\lambda+1} f(z)-(\lambda+2) D^{\lambda} f(z), \quad \lambda>-1
$$

For $\lambda=n \in N_{0}=\{0,1,2 \ldots \ldots\}$ we note that the relation (1.3) may be expressed as

$$
D^{n} f(z)=\frac{1}{z}\left(\frac{z^{n+1} f(z)}{n !}\right)^{(n)}
$$




$$
D^{\lambda} f(z)=\frac{1}{z}+\sum_{k=1}^{\infty} B_{k}(\lambda) a_{k} z^{k}
$$

where

$$
B_{k}(\lambda)=\frac{(\lambda+1)(\lambda+2)(\lambda+3) \ldots(\lambda+k-1)}{(k+1) !}
$$

A function

$$
f(z)=\frac{1}{z}+\sum_{k=1}^{\infty}\left|a_{k}\right| z^{k} \in M
$$

is said to be in the class $M^{*}(\alpha, \lambda, A, B)$ if it satisfies the condition (1.3) with $-1 \leq B \leq 0$.

The main object of this paper is to present a systematic investigation of various properties of the general class $M^{*}(\alpha, \lambda, A, B)$ and to determine neighborhood results and partial sums for the general class of meromorphic univalent functions with positive coefficients.

\section{Properties of the class $M^{*}(\alpha, \lambda, A, B)$}

Theorem 1. Let $f(z)=\frac{1}{z}+\sum_{k=1}^{\infty}\left|a_{k}\right| z^{k}$ be analytic and univalent in U. Then $f(z) \in$ $M^{*}(\alpha, \lambda, A, B)$ if and only if

$$
\sum_{k=1}^{\infty} \frac{k(1-B \alpha)}{\alpha(A-B)} B_{k}(\lambda)\left|a_{k}\right| \leq 1 .
$$

The result is sharp for the function $f(z)$ given by

$$
f(z)=\frac{1}{z}+\frac{\alpha(A-B)}{k B_{k}(\lambda)(1-B \alpha)} z^{k} .
$$


Proof. Let $f(z)=\frac{1}{z}+\sum_{k=1}^{\infty}\left|a_{k}\right| z^{k} \in M^{*}(\alpha, \lambda, A, B)$. Then

$$
\left|\frac{z^{2}\left(D^{\lambda} f(z)\right)^{\prime}+1}{B z^{2}\left(D^{\lambda(z)}\right)^{\prime}+A}\right|<\alpha
$$

Substituting for $\left(D^{\lambda} f(z)\right)^{\prime}$, we get

$$
\left|\frac{z^{2}\left(\frac{-1}{z^{2}}+\sum_{k=1}^{\infty} k B_{k}(\lambda) a_{k} z^{k-1}\right)+1}{B z^{2}\left(\frac{-1}{z^{2}}+\sum_{k=1}^{\infty} k B_{k}(\lambda) a_{k} z^{k-1}\right)+A}\right|<\alpha
$$

That is,

$$
\left|\frac{\sum_{k=1}^{\infty} k B_{k}(\lambda) a_{k} z^{k+1}}{(A-B)+\sum_{k=1}^{\infty} B k B_{k}(\lambda) z^{k+1}}\right|<\alpha
$$

Since $|\operatorname{Re}(z)| \leq|z|$ for any $z$, choosing values of $z$ to be real, (2.3) yields

$$
\sum_{k=1}^{\infty} k(1-B \alpha) B_{k}(\lambda)\left|a_{k}\right| \leq \alpha(A-B)
$$

On other hand if we let $z \in \partial E$, then we find that

$$
\left|\frac{z^{2}\left(D^{\lambda} f(z)\right)^{\prime}+1}{B z^{2}\left(D^{\lambda} f(z)\right)^{\prime}+A}\right| \leq \frac{\sum_{k=1}^{\infty} k B_{k}(\lambda) a_{k}}{(A-B)+\sum_{k=1}^{\infty} B k B_{k}(\lambda)}<\alpha
$$

Finally, by observing that the function $f(z)$ given by $(2.2)$ is indeed an extremal function for the assertion (2.1), which completes the proof of Theorem 1. 
The following theorem is an easy consequence of Theorem 1 .

Theorem 2. Let each of function $f_{j}(z)$ defined by

$$
f(z)=\frac{1}{z}+\sum_{k=1}^{\infty}\left|a_{k, i}\right| z^{k} .(i=1,2,3 \ldots)
$$

be in the class $M^{*}(\alpha, \lambda, A, B)$. Then the furiction $h(z)$ defined by

$$
h(z)=\sum_{i=1}^{\infty} \mu_{i} f_{i}(z) \quad\left(\mu_{i} \geq 0 \text { and } \sum_{i=1}^{\infty} \mu_{i}=1\right)
$$

is also in the class $M^{*}(\alpha, \lambda, A, B)$.

Theorem 3. Let $f(z) \in M^{*}(\alpha, \lambda, A, B)$ then we have $f(z)$ is meromorphically starlike of order $\delta$ in $|z|<r$, that is

$$
\begin{aligned}
& \operatorname{Re}\left\{\frac{-z f^{\prime}(z)}{f(z)}\right\}>\delta,\left(|z|<r_{1} \text {, when } 0 \leq \delta<1\right) \\
& r_{1}=\inf _{k \geq 1}\left\{B_{k}(\lambda) \frac{k(1-\delta)(1-\alpha B)}{\alpha(A-B)(k+\delta)}\right\}^{\frac{1}{k+1}}
\end{aligned}
$$

The result is sharp for the function $f(z)$ given by (2.2).

Proof. From Theorem 1, we have

$$
\sum_{k=1}^{\infty} \frac{k+\delta}{(1-\delta)}\left|a_{k}\right| z^{k+1}<\sum_{k=1}^{\infty} B_{k}(\lambda) \frac{k(1-\alpha B)}{(A-B) \alpha}\left|a_{k}\right| \leq 1\left(|z|<r_{1}\right)
$$

Therefore for $|z|<r_{1}$

$$
\begin{aligned}
\left|\frac{\frac{z f^{\prime}(z)}{f(z)}+1}{\frac{z f^{\prime}(z)}{f(z)}-(1-2 \delta)}\right| & =\left|\frac{\sum_{k=1}^{\infty}(k+1) a_{k} z^{k}}{2(1-\delta)-\sum_{k=1}^{\infty}(k-(1-2 \delta)) a_{k} z^{k}}\right| \\
& =\frac{\sum_{k=1}^{\infty}(k+1) B_{k}(\lambda)\left|a_{k} \| z\right|^{k+1}}{2(1-\delta)-\sum_{k=1}^{\infty}(k-1+2 \delta)\left|a_{k} \| z\right|^{k+1}}<1
\end{aligned}
$$

which implies that (2.6) is true. 


\section{Neighborhoods}

The concept of neighborhood of analytic functions was first introduced by Goodman [4] and then generalized by Ruscheweyh [9]. In this section we shall extend the concept of neighborhoods to meromorphic univalent functions.

For $\delta \geq 0,-1 \leq B<A \leq 1$ and $f(z)=\frac{1}{z}+\sum_{k=1}^{\infty} a_{k} z^{k} \in M$. We define neighborhood of $f(z)$ by

$$
\begin{aligned}
N_{\delta}(f) & =N_{\delta}(f, \alpha, \lambda, A, B) \\
& =\left\{g(z)=\frac{1}{z}+\sum_{k=1}^{\infty} a_{k} z^{k} \in M: \sum_{k=1}^{\infty} \frac{(k(1-|B| \alpha))}{\alpha(A-B)} B_{k}(\lambda)\left|b_{k}-a_{k}\right| \leq \delta\right\}
\end{aligned}
$$

Theorem 4. Let $\delta>0$ and $\alpha>0$. If $f(z)=\frac{1}{z}+\sum_{k=1}^{\infty} a_{k} z^{k} \in M$ satisfies

$$
\frac{f(z)+\varepsilon z^{-1}}{1+\varepsilon} \in M^{*}(\alpha, \lambda, A, B)
$$

for any complex number $\varepsilon$ such that $|\varepsilon|<\delta$, then $N_{\delta}(f) \subset M^{*}(\alpha, \lambda, A, B)$.

Proof. It is easily seen from (1.2) that $g(z) \in M^{*}(\alpha, \lambda, A, B)$ if and only if for any complex number $\sigma$ with $|\sigma|=1$

$$
\left|\frac{z^{2}\left(D^{\lambda} g(z)\right)^{\prime}+1}{B z^{2}\left(D^{\lambda} g(z)\right)^{\prime}+A}\right| \neq \sigma \alpha \quad(z \in U)
$$

which is equivalent to

$$
\frac{g(z)^{*} h(z)}{z^{-1}} \neq 0 \quad(z \in E)
$$

where

$$
h(z)=\frac{1}{z}+\sum_{k=1}^{\infty} c_{k} z^{k}=\frac{1}{z}+\sum_{k=1}^{\infty} \frac{(k-1)(1-B \sigma \alpha)}{\alpha \sigma(B-A)} B(\lambda) z^{k-1}
$$


From (3.3) we have

$$
\left|c_{k}\right|=\left|\frac{(k-1)(1-B \sigma \alpha)}{\sigma \alpha(B-A)} B_{k}(\lambda)\right| \leq \frac{(k-1)(1+|B| \alpha)}{\sigma \alpha(A-B)} B_{k}(\lambda)
$$

If $f(z)$ of the form (1.1) and belonging to the class $M$ satisfies the condition (3.1) and (3.2) yields

$$
\begin{aligned}
& \left|\frac{f(z)^{*} h(z)}{z^{-1}}\right| \geq \delta \quad(z \in E) \\
& \text { Now we let } p(z)=\frac{1}{z}+\sum_{k=1}^{\infty} b_{k} z^{k} \in N_{\delta}(f) \text {, then so that } \\
& \left|\frac{(\rho(z)-f(z)))^{*} h(z)}{z^{-1}}\right|=\left|\sum_{k \neq 1}^{\infty}\left(b_{k}-a_{k}\right) c_{k} z^{k}\right| \\
& \leq|z| \sum_{k=1}^{\infty} \frac{k(1-|B| \alpha)}{\alpha(A-B)} B_{k}(\lambda)\left|\left(b_{k}-a_{k}\right)\right|<\delta
\end{aligned}
$$

Thus for any complex number $\sigma$ such that $|\sigma|=1$, we have

$$
\frac{p(z)^{*} h(z)}{z^{-1}} \neq 0(z \in E)
$$

which implies that $p(z) \in M^{*}(\alpha, \lambda, A, B)$. The proof is complete.

In the following theorem we obtain partial sums for the general class of meromorphic univalent functions with positive coefficients.

Theorem 5. Let $f \in M$ be given by (1.1) and define the partial sums $s_{1}(z)$ and $s_{n}(z)$ by

$$
s_{1}(z)=\frac{1}{z} \quad \text { and } \quad s_{n}(z)=\frac{1}{z}+\sum_{k=1}^{n-1} a_{k} z^{k}(n \in N \backslash\{\}) \text {. }
$$


Suppose also that

$$
\sum_{k=1}^{\infty} d_{k}\left|a_{k}\right| \leq l,
$$

where

$$
d_{k}:=\frac{k(1-|B| \alpha)}{(B-A)} B_{k}(\lambda)
$$

Furthermore,

$$
\operatorname{Re}\left\{\frac{f(z)}{s_{n}(z)}\right\}>1-\frac{1}{d_{n}}(z \in U, n \in N)
$$

and

$$
\operatorname{Re}\left\{\frac{s_{n}(z)}{f(z)}\right\}>\frac{d_{n}}{1+d_{n}}(z \in U, n \in N)
$$

Each of the bounds in (3.6) and (3.7) is the best possible for $n \in N$.

Proof. It is easily seen that $\frac{1}{z} \in M(\alpha, \lambda, A, B)$. Thus from Theorem 4 of the hypothesis (3.5) we have

$$
N_{1}\left(\frac{1}{z}\right) \subset M(\alpha, \lambda, A, B)
$$

which shows that $f \in M \cdot(\alpha, \lambda, A, B)$ as asserted by Theorem 5 .

Next for the coefficient $d_{k}$ given by (3.5), it is not difficult to verify that

$$
d_{k+1}>d_{k}>1(k=1,2,3, \ldots)
$$

Therefore, we have

$$
\sum_{k=1}^{n-1}\left|a_{k}\right|+d_{n} \sum_{k=n}^{\infty}\left|a_{k}\right| \leq \sum_{k=1}^{\infty} d_{k}\left|a_{k}\right| \leq 1
$$


by using the hypothesis (3.5) again by setting,

$$
\begin{aligned}
g_{1}(z) & =d_{n}\left\{\frac{f(z)}{s_{n}(z)}-\left(1-\frac{1}{d_{n}}\right)\right\} \\
& =1+\frac{d_{n} \sum_{k=n}^{\infty} a_{k} z^{k}}{1+\sum_{k=1}^{n=1} a_{k} z^{k}}
\end{aligned}
$$

and applying (3.10), we find that

$$
\left|\frac{g_{1}(z)-1}{g_{1}(z)+1}\right| \leq \frac{d_{n} \sum_{k=0}^{\infty}\left|a_{k}\right|}{2-2 \sum_{k=1}^{n-1}\left|a_{k}\right|-d_{n} \sum_{k=1}^{\infty}\left|a_{k}\right|} \leq 1, \quad z \in U,
$$

which readily yields the assertion (3.6) of Theorem 5 .

If we take

$$
f(z)=\frac{i}{z}-\frac{z^{n}}{d_{n}}
$$

then

$$
\frac{f(z)}{s_{n}(z)}=\frac{\frac{1}{z}-\frac{z^{n}}{d_{n}}}{\frac{1}{z}+\sum_{k=1}^{n-1} a_{k} z^{k-1}}=1-\frac{z^{n}}{d_{n}} \rightarrow 1-\frac{1}{d_{n}} \text { as } z \rightarrow 1,
$$

which shows that the bound in (3.7) is the best possible for each $n \in N$. Similarly, we put

$$
\begin{aligned}
g_{2}(z) & =\left(1+d_{n}\right)\left\{\frac{s_{n}(z)}{f(z)}-\frac{d_{n}}{1+d_{n}}\right\} \\
& =1-\frac{\left(1+d_{n}\right) \sum_{k=n}^{\infty} a_{k} z^{k}}{1+\sum_{k=1}^{\infty} a_{k} z^{k}}
\end{aligned}
$$


and making use of (3.10), we can deduce that

$$
\left|\frac{g_{2}(z)-1}{g_{2}(z)+1}\right| \leq \frac{\left(1+d_{n}\right) \sum_{k=n}^{\infty}\left|a_{k}\right|}{2-2 \sum_{k=1}^{n-1}\left|a_{k}\right|+\left(1-d_{n}\right) \sum_{k=n}^{\infty}\left|a_{k}\right|} \leq 1
$$

which leads us immediately to the assertion (3.7) of Theorem 5.

The bound in (3.7) is sharp for each $n \in N$, with the extremal function $f(z)$ given by (3.13). The proof of the Theorem is thus completed.

\section{References}

1. M.K. Aouf, On a certain class of meromorphic univalent functions with positive coefficients, Rent. Math., (7) 11 (1991), 209-219.

2. S.K. Bajpai, A note on a class of meromorphic univalent functions, Rev. Roumaine Math. Pure \& Appl., 22 (1977), 295 - 297.

3. J. Clunie, On meromorphic Schlicht function, J. London Math. Soc., 34 (1959), 215 - 216.

4. A.W. Goodman, Univalent functions and nonanalytic curves, Proc. Amer. Math. Soc., 8 (1957), 598 - 601 .

5. J.L. Liv and H.M. Srivastava, A linear operator and associated families of meromorphically multivalent functions, J. Math. Anal \& Appl,, 259 (2001), 566 - 581.

6. J.L. Liu, Properties of some families of meromorphic p-valent functions, Math Japonica., 52 No: $3(2000), 425-434$.

7. Ch. Pommerenke, On meromorphic starlike functions, Pacific J. Math., 13 (1963), $212-235$.

8. O. Sangkwon and N.E. Cho, Hadamard product of certain meromorphic univalent functions, Tamkang J. Math., Vol. 28, No. 4 Winter 1997.

9. S. Ruscheweyh, Neighborhoods of univalent functions, Proc. Amer. Math. Soc., 81 (1981), 521.527. 